\title{
La réception du 18 Brumaire en Helvétie
}

\author{
Alain-Jacques Czouz-Tornare
}

\section{(2) OpenEdition}

\section{Journals}

Édition électronique

URL : https://journals.openedition.org/ahrf/298

DOI : 10.4000/ahrf.298

ISSN : $1952-403 X$

Éditeur :

Armand Colin, Société des études robespierristes

Édition imprimée

Date de publication : 1 décembre 1999

Pagination : 727-745

ISSN : 0003-4436

Référence électronique

Alain-Jacques Czouz-Tornare, «La réception du 18 Brumaire en Helvétie », Annales historiques de la Révolution française [En ligne], 318 | octobre-décembre 1999, mis en ligne le 11 avril 2006, consulté le 24 avril 2022. URL : http://journals.openedition.org/ahrf/298 ; DOI : https://doi.org/10.4000/ahrf.298

Ce document a été généré automatiquement le 24 avril 2022.

Tous droits réservés 


\title{
La réception du 18 Brumaire en Helvétie
}

\author{
Alain-Jacques Czouz-Tornare
}

1.La détresse d'une Suisse en plein désarroi

1 Figé dans des structures archaïques qui ne lui permettaient pas d'évoluer, le Corps Helvétique s'effondre piteusement en 1798. Jusqu'au printemps 1798, il n'existait de pouvoir central qu'au sein des cités-États et les différentes composantes de ce qu'on appelait la Suisse n'étaient unies que par une alliance aux termes de laquelle les cantons souverains se garantissaient réciproquement leurs libertés, leur indépendance, et leur sécurité envers l'étranger, ainsi que l'ordre à l'intérieur et le droit de chacun de dominer individuellement ou en commun certains territoires. C'est la Révolution helvétique de 1798 qui établit un réel État de droit et les bases d'une Suisse nouvelle. Le passage obligé par le centralisme fut paradoxalement la condition sine qua non à l'établissement d'un État fédératif. L'irruption révolutionnaire puis l'invasion française ${ }^{1}$ furent vécues comme un traumatisme par une grande partie des élites dirigeantes. L'occupation française fit éclater au grand jour l'état d'inféodation dans lequel se trouvaient les ligues suisses, qui se virent brusquement imposer un régime centralisé contraire aux traditions ancestrales du pays. La constitution de la République Helvétique une et indivisible fut promulguée à Aarau le 12 avril 1798 et le Directoire helvétique installé le 19 avril.

Des Suisses furent directement impliqués dans le coup d'État du 18 Brumaire, derrière lequel se dissimulent quelques banquiers helvétiques. Comptant parmi les principaux artisans de la journée du 18, Perregaux se retrouve sénateur le 4 Nivôse an VIII (25 décembre 1799) avant de devenir l'organisateur de la Banque de France - créée en 1700 - dont il occupera le premier fauteuil de régent et celui de président du Conseil de Régence ${ }^{2}$.

3 Lorsque survient la journée du 18 Brumaire, une partie de la Suisse sert de champ de bataille aux grandes puissances et se trouve encore occupée par les forces de la Coalition. Selon Johann Rudolf Dolder (1753-1807), alors président du Directoire helvétique, les trois-quarts des Suisses étaient dans la misère ${ }^{3}$. Comme l'exprime le 
Directeur Frédéric-César Laharpe (1754-1838) ${ }^{4}$ dans une motion du 4 novembre 1799 : "Le séjour de deux armées sur notre territoire a réduit notre république à la dernière extrémité. Notre existence comme nation, notre liberté, notre indépendance, nos vies et nos fortunes sont réduites à attendre humblement au vainqueur de prononcer sur nos destinées ${ }^{5}$. Dans sa lettre à Sieyès, du 6 juin 1799, le patriote vaudois espère que "Le gouvernement français n'a jamais voulu sans doute que notre révolution fût acquise aux dépens de notre existence ${ }^{6}$. Et d'ajouter le 5 juillet, dans une autre lettre à Sieyès : " Notre détresse est extrême, et si elle se prolonge l'Helvétie est ruinée. " ${ }^{7}$. Comme le remarque M.-C. Jéquier : «Les soulèvements réactionnaires, les résistances multiples au nouvel ordre des choses, les nostalgiques de l'ancien compliquent encore une situation déjà catastrophique ${ }^{8}$. Les relations entre le Directoire français et son homologue helvétique étaient particulièrement tendues, même si l'arrivée de Sieyès au Directoire à la place du redouté Reubell, en mai 1799, avait fait naître les plus grands espoirs. Mais il semble bien que le Directoire français ne maîtrisa pas la situation et que les militaires agissaient bien souvent à leur guise. De l'aveu même du Directoire helvétique, la plus grande incertitude planait sur ses relations avec la France ${ }^{9}$, le Directoire helvétique réfractaire risquant de se faire "cisalpiniser" par le général Masséna aux dires de La Harpe ${ }^{10}$, qui déplorait «la politique bornée du Directoire » français ${ }^{11}$. Masséna, « connu pour sa rapacité » selon B. Nabonne ${ }^{12}$ se vit gratifier par le doyen Bridel du mot suivant : "Je l'enrichis d'un $t$ pour qu'étant Massénat, son nom rime avec Rapinat ${ }^{13}$. Après le retrait de Reubell, le 16 mai 1799, l'attitude française est pourtant à l'apaisement. Le chargé d'affaire français Henri-François Perrochel, plutôt conciliant, se fait l'écho des perpétuelles doléances du gouvernement suisse. Ainsi écrit-il à Talleyrand, le 8 messidor (27 juin 1799) : «Au reste, citoyen ministre, il s'est commis, depuis si longtemps, dans ce pays-ci des actes tellement propres à nous aliéner pour jamais l'esprit de la nation, que ce que nous pouvons y ajouter sera désormais fort peu sensible ${ }^{14}$. Selon Alfred Rufer, décrivant l'affligeante situation de l'Helvétie en 1799 : «Le Directoire qui n'était pas même en état de venir en aide au peuple français ne pouvait rien faire en faveur de son allié " ${ }^{15}$. En cet automne 1799, la misère et l'épuisement de la Suisse étaient tels que le désespoir touchait toutes les couches de la population.

2. La « métamorphose " ${ }^{16}$ de la République française vue par Mallet du Pan

4 Un observateur aussi attentif que le Suisse Jacques Mallet du Pan ${ }^{17}$ ne pouvait ignorer l'impasse dans laquelle se trouvait le Directoire sur sa fin. Ainsi note-t-il, de Londres, en date du 25 août 1799 : « La dictature la plus compacte, la plus absolue, la moins exposée aux conflits des juridictions et aux contrariétés, ne serait pas de trop dans un pays où, depuis Brest à Perpignan un abîme de troubles, de discordes et d'anarchie s'ouvre et s'approfondit" ${ }^{18}$. Selon lui, "la République se traînait entre un gouvernement chancelant et une Législature anarchique» ${ }^{19}$. Traitant Bonaparte tour à tour d'« Hercule » et de "charlatan du Pont-neuf », il n'en perçoit pas moins l'importance du personnage au moment même où le général triomphe à Paris : "L'arrivée de Bonaparte n'est point, à beaucoup près, un événement indifférent. Son génie, son ambition de gloire, ses talents, sa réputation, ont un poids dans les destinées de la République ${ }^{20}$. Le 18 Brumaire laisse le publiciste genevois perplexe. Pour lui cette «dernière révolution » du 10 novembre stoppe net le processus révolutionnaire ${ }^{21}:$ Il qualifie ainsi la nouvelle constitution du 22 frimaire an VIII « d'apostasie solennelle des maximes adoptées en 1789 " ${ }^{22}$. "S'il y a continuité d'usurpation, il n'y a sûrement pas continuité du régime antérieur. » Et de relever à titre d'exemple que « la liberté de la 
presse, ce palladium de la révolution et de la république, n'existe plus qu'à la discrétion du gouvernement ${ }^{23}$. Dans son ultime numéro du 25 mars 1800, Mallet du Pan, à bout de forces mais toujours lucide, observe que "nous avons une monarchie sans dynastie » et que l'on s'oriente déjà vers un pouvoir héréditaire ${ }^{24}$.

D'un point de vue suisse, Mallet du Pan observe que «la majorité du Corps législatif a profité de la révolution survenue à Paris au mois de novembre, pour opérer aussi un déménagement politique ». Et de percevoir déjà l'ébauche d'une contre-révolution au travers de ces hommes politiques suisses qui «s'avouent la nécessité de dissoudre en entier l'échafaudage élevé par la révolution, de rétablir l'indépendance et la souveraineté de chaque canton dans tout ce qui ne touche point aux intérêts généraux de l'État, et de composer un corps fédératif plus compact, plus puissant, plus réactif que ne le fut celui d'autrefois. " ${ }^{25}$

3. Les retombées du 18 Brumaire en Suisse

6 Le 10 décembre 1799, le ministre de la Police informe le Directoire exécutif de l'influence sur « l'esprit public du canton de Lucerne », des « changements survenus en France le 18 Brumaire »: "Toujours le peuple a envisagé le gouvernement helvétique comme dépendant de celui de la France [...] La considération dont le gouvernement devrait jouir en est singulièrement diminuée. Et l'opinion défavorable qui en naît s'étend sur tout ce qui y est relatif. Les changements opérés en France en laissent supposer aussi en Helvétie. Ils sont même généralement désirés. L'opinion publique s'est prononcée sur notre constitution qui paraît au peuple trop compliquée, trop dispendieuse et aucunement conforme à ses besoins. Ses souffrances augmentent encore son mécontentement. Les réquisitions excessives en denrées, en bétail, en hommes, les logements militaires, les vexations qu'il en éprouve fatiguent et épuisent le peuple [...]. Je ne vous dissimule pas, citoyen Directeur, que la situation actuelle des choses me paraît très inquiétante, les dangers augmentent à raison du découragement des fonctionnaires chargés de veiller à la sûreté publique. L'état d'incertitude qui tourmente chaque citoyen fait perdre de vue l'intérêt général [...] L'état de révolution qui a été entretenu en France pendant quelques années a dégradé infiniment la moralité du peuple, et si encore les lumières et les connaissances ont surnagé au vandalisme qu'on s'est efforcé d'y établir, c'est qu'elles ont été répandues sur la surface de ce grand empire avec une telle abondance qu'elles résistaient par leur propre force au choc qui aurait dû les anéantir. Mais en Helvétie où les mœurs ne sont qu'une suite d'habitudes et l'effet des opinions religieuses, où l'industrie, le commerce, les lumières n'ont point encore obtenu ni de consistance, ni d'étendue, ni de force, la prolongation d'un état révolutionnaire ne peut que nous replonger dans une barbarie qui anéantira toute moralité, toutes les cultures, tout moyen de nous relever de l'état de dégradation, qui nous exposerait à devenir le mépris et le jouet des peuples qui nous entourent. " ${ }^{26}$

7 Tous les partis en Suisse accueillirent ou firent semblant d'accueillir le 18 Brumaire avec soulagement. À commencer par les adversaires de la Révolution, comme a pu le constater É. Dunant : "L'influence du coup d'État du 18 Brumaire se fit sentir tôt après dans les conseils législatifs de la République helvétique. Dès lors le parti républicain modéré auquel s'étaient ralliés certains membres des familles patriciennes, conçut l'espoir d'être à l'avenir soutenu par les Consuls de la République française " ${ }^{27}$. Ce que confirme Louis André Pichon ${ }^{28}$, secrétaire de l'ambassade de France, dans un envoi à Reinhard du 26 brumaire an VIII : «À la suite des changements survenus en France le 18 Brumaire le parti des anciens gouvernants a espéré trouver un appui dans les 
Consuls " ${ }^{29}$. À l'opposé, les responsables politiques helvétiques virent tout d'abord dans le 18 Brumaire l'occasion inespérée de donner un nouveau départ, un second souffle, au régime issu du révolutionnement de la Suisse l'année précédente. Comme l'exprime Frédéric-César La Harpe dans une lettre à son ancien élève l'empereur Alexandre ler, du ler octobre 1801: "La chute du Directoire jacobin et l'avènement de Bonaparte relevèrent dans ce moment l'espoir des amis de la patrie qui, dupes de leur cœur, se persuadèrent trop légèrement que l'heure du soulagement et de la générosité avait sonné » ${ }^{30}$. Le 15 novembre 1799 (24 brumaire an VIII), Urs Peter Josef Ludwig Zeltner (1765-1830) ${ }^{31}$, chargé d'affaires de la République helvétique à Paris de 1798 à 1802, prend l'initiative de se réjouir du changement de régime dans un envoi au ministre Charles Frédéric Reinhard ${ }^{32}$ : «Des lumières profondes, l'amour de la justice et une vertu guerrière sans exemple, joints au respect pour l'indépendance des peuples alliés, sont les qualités de vos Consuls; ils promettent à l'Helvétie les vrais rapports politiques fondés sur le bonheur des deux nations toujours amies, malgré les commotions qui les ont quelquefois agitées. Le gouvernement français fera enfin jouir l'Helvétie de tout ce qu'elle connaît de plus précieux, de sa liberté et de son indépendance. Le respect dû aux traités va remplacer ce système de violation et vexation qui pèse depuis trop longtemps sur ce malheureux pays. Intimement convaincu, citoyen ministre, que tels seront pour ma patrie les heureux résultats de cette grande mesure, je ne puis qu'en féliciter les auteurs et faire des vœux pour le prompt accomplissement du but qu'ils se proposent, le bonheur et la paix pour la République française et ses fidèles alliés. Veuillez faire agréer aux Consuls l'expression de ces sentiments et les assurer qu'ils seront partagés par le gouvernement et le peuple helvétiqu. " ${ }^{33}$. Le Directoire helvétique réagit aussitôt et charge le 18 novembre, Jenner ${ }^{34}$ son ex-ministre à Paris de "faire toutes les démarches propres à fixer la pensée du gouvernement français en faveur de l'Helvétie, à resserrer les liens qui unissent les deux peuples, et à donner à leurs relations respectives une solidité respectable ». De Lausanne, le 16 novembre, le patriote vaudois Henri Monod (1754-1833) se réjouit de ce qui vient de se passer dans une lettre à son ami Laharpe : "Voici des événements mon cher, qui vont occasionn[er beaucoup de] remue-ménage, j'en augure bien pour nous. Ce qui me fâche, c'est que tout dépend de l'existence de deux hommes (Bonaparte et Sieyès), et qu'ainsi la fortune publique est de nouveau remise à la fortune. J'ose croire cependant qu'ils vivront, et que cet homme extraordinaire ne voudra pas se diffamer. Quel beau rôle si, après avoir été un César à la tête des armées, il sait profiter de sa gloire et de ses avantages pour jouer le contraire du rôle du Romain à la tête de la République en donnant à sa patrie la liberté que César ôta à la sienne " ${ }^{35}$. Pour Laharpe, soutenu il y a encore peu par Reubell et qui a ses entrées auprès de Bonaparte qui a été le compagnon d'armes d'Amédée Laharpe, l'occasion est inespérée de faire passer ses idées. Lui-même avait imaginé « des mesures de salut public " à l'encontre des Conseils qu'il désirait déjà dissoudre, comme la constitution d'ailleurs lui en donnait le pouvoir. Les 7-8 novembre 1799, au moment même où Bonaparte s'apprête à passer à l'action, Laharpe écrit à son ami Monod: «Mon parti est pris. Je reconnais l'impossibilité de sauver mon pays avec des législateurs qui n'ont ni ensemble, ni sentiment de leur dignité ou des périls que court la chose publique " ${ }^{36}$. Dès le 19 novembre, il écrit au Consul, en lui exprimant son désir " de voir les républiques française et helvétique consolidées et sincèrement unies " ${ }^{37}$. Pour le cofondateur de la République helvétique, celle-ci ne peut être réellement utile à la Grande Nation que si elle est neutre, libérée d'une trop voyante tutelle française et dotée d'institutions républicaines fortes ${ }^{38}$. Le 11 décembre 1799, Laharpe écrit sans 
ambages au Consul Bonaparte: "Vous convient-il de nous laisser dissoudre? Vous convient-il d'employer soixante mille hommes à garder notre territoire, lorsque nous pourrions en fournir une partie, si nous étions mieux organisés? Vous convient-il de courir pour la seconde fois la chance à laquelle vous avez échappé comme par miracle, celle de voir la moitié de l'Helvétie vendéisée par les Autrichiens? Citoyen Consul ! Je ne veux point pénétrer les mystères politiques du gouvernement français, mais il me semble que s'il lui convient que l'Helvétie continue à former un État indépendant (et je pense qu'il n'est qu'un ennemi de la France qui puisse soutenir l'avis opposé), il me semble, dis-je : qu'il doit tout faire pour que la République helvétique se consolide, s'organise très promptement et devienne assez forte pour pouvoir lui servir de poste avancé. Il me semble que le gouvernement français doit soutenir, et vigoureusement, le Directoire helvétique, l'aider par son influence à réformer très vite les vices de l'organisation actuelle et lui assurer la force qui lui manque. » ${ }^{39}$

Les Suisses pensaient pouvoir compter sur Sieyès qui, selon Jenner, convenait « qu'on a agi contre l'intérêt de la France en faisant sortir la Suisse de sa neutralité » et recevait " avec intérêt et empressement tous les moyens qui pourraient nous faire rentrer dans cet état heureux " ${ }^{40}$. Laharpe avait bien compris l'erreur commise par le Directoire français qui, en imposant sans discussion une alliance offensive le 19 août 1799, avait corollairement privé la France du bouclier de la neutralité suisse qui préservait de toute invasion les départements français de Genève à Bâle. Laharpe devra rapidement déchanter car Sieyès, à qui il a adressé un mémoire circonstancié le 7 décembre ${ }^{41}$, est évincé le 12 déjà et le nouveau chargé d'affaires français par intérim en Suisse, Pichon, "successeur de Perrochel ${ }^{42}$ et créature de Talleyrand », selon Laharpe ${ }^{43}$, " circonvenu par les républicains modérés, dénonce systématiquement les initiatives de Laharpe ${ }^{44}$ et favorise dans le sein des Conseils l'élément dit modéré en attendant le remplacement du Directoire helvétique par une autorité provisoire. Toutefois comme le relève Étienne Hofmann : «Même sans cela, il est peu probable que Bonaparte eût écouté le Directeur helvétique; il a trop besoin en Suisse d'un personnel politique plus souple, moins ombrageux. Si des autorités plus modérées s'installaient à Berne, cela conviendrait davantage à sa diplomatie: il vaut mieux en effet ne pas trop irriter l'adversaire autrichien par une politique trop agressive en Suisse ${ }^{45}$. Or, Laharpe reste un idéaliste dans une époque qui requiert beaucoup de souplesse idéologique : "Jacobin, Laharpe l'ait par son énergique confiance dans le rôle du pouvoir et de la centralisation politique, par le goût pour la vertu républicaine, qu'il incarne jusqu'à la caricature, par l'inflexibilité et la droiture toute romaine de son caractère " ${ }^{46}$. Pour Talleyrand, des Laharpe et Ochs ne sont que des "brouillons suisses" ${ }^{47}$. Selon Pichon, le parti oligarchique regroupant les membres des anciennes familles dirigeantes est celui « qui, à la longue doit prévaloir et auquel nous devons nous attacher ${ }^{48}$. Il faut tout de même relever ici que durant toute la Révolution, les Français avaient recherché la collaboration des patriciens suisses au pouvoir qui appartenaient à la clientèle traditionnelle de la France. À ce titre, le Club helvétique ${ }^{49}$ de Paris fut bien plus un outil de pression à leur égard qu'un véritable instrument de prise de pouvoir. Aucun de ses membres n'accédera à des fonctions importantes après l'écroulement de l'Ancien Régime en Suisse. L'avocat Castella, ancien chef du Club, dut se contenter du poste de sous-préfet de Gruyère dans le canton de Fribourg. Quelques patriciens, comme le Fribourgeois Jean de Montenach, premier président de la Municipalité de Fribourg, acceptèrent de jouer le jeu, mais la plupart refusèrent de s'engager auprès de la France révolutionnaire, contraignant le Directoire à faire appel à des notables considérés 
comme beaucoup moins représentatifs. Au fond, il ne tenait qu'aux anciens gouvernants de retrouver le pouvoir pour autant qu'ils veuillent bien s'aligner sur la France. Or, les anciens dirigeants persistaient obstinément à jouer la carte de la seconde coalition. C'est faute de mieux et presqu'à contre-cœur que le Directoire puis le Consulat continuèrent à utiliser le personnel révolutionnaire. Tous les efforts de Napoléon Bonaparte et de son entourage tendirent à convaincre les patriciens les moins récalcitrants à se rallier à son programme de restauration partielle.

Le 21 novembre (30 brumaire), Pichon notifie l'installation de la commission consulaire exécutive au gouvernement helvétique qu'il chercha à rassurer en espérant que ce dernier voit dans ce changement «des motifs pour concourir avec plus d'empressement que jamais à dissiper les nuages qui ont un moment inspirés des inquiétudes aux amis de l'Helvétie et relever les espérances de ses ennemis " ${ }^{50}$. Le 27 novembre, le ministre helvétique des Affaires étrangères répond en ces termes à l'envoi de Pichon: "La Révolution du 18 Brumaire, sanctionnée par la loi du 19, que vous voulez bien nous communiquer, en rajeunissant et replaçant sur l'aplomb de ses primitives bases la république chancelante, a justifié la haute opinion que les fidèles alliés de la nation française conçurent de son caractère, lorsqu'ils se jetèrent sous l'égide de ses vertus. Elle a ravivé leur confiance, épuré l'horizon assombri de la liberté et ouvert à l'Europe une sublime perspective, à laquelle on ne croyait plus, parce que trop souvent elle fut promise, sans les moyens de la réaliser. Aujourd'hui, ces moyens existent, l'heureux accord de la sagesse, du génie et de l'héroïsme, cimenté et célébré par l'opinion publique, nous garantit les succès des travaux du gouvernement provisoire de la France. Les engagements de ses législateurs, de ses consuls seront remplis. Notre attente ne sera pas vaine. Les espérances d'un grand peuple qui dépose en leurs mains ses intérêts les plus chers, celles des États qui s'associent à ses destinées, ne seront pas déçues. Lorsque vous présentez aux autorités helvétiques la notification d'un événement si mémorable, vous leur offrez, citoyens, un gage précieux, mais non imprévu, des intentions pures qui animent les nouveaux magistrats de la République française. Il était de la dignité de votre gouvernement régénéré d'indiquer à la fois, par ce prompt témoignage de sa bienveillance, et son regret des illusions qui furent momentanément la source des plus pénibles alarmes, et sa ferme volonté d'établir désormais la garantie de nos droits sur des principes stables [...] Le beau jour qui a fait luire sur la France un ordre de chose dont la justice et la vraie liberté constituent la base, nous annonce avec certitude que le peuple qui devint votre allié par la nature, par affection et par la foi des traités, n'invoquera plus en vain ces garants de son existence. " 51

10 Les Suisses désiraient par dessus tout négocier un arrangement conforme aux principes de l'article III du traité d'alliance ${ }^{52}$. Le Directoire helvétique cherchait à renégocier le traité d'alliance de septembre 1798 en rétablissant la neutralité suisse, et la convention de Lucerne du 30 novembre 1798 sur la levée du corps auxiliaire, en déliant l'Helvétie de l'engagement de fournir 18000 hommes. Jenner reçut pour instructions de ne négliger «aucun moyen de préparer l'admission d'un plénipotentiaire helvétique au congrès pour la paix » et de "représenter de la manière la plus forte l'état malheureux de l'Helvétie ", en insistant notamment sur les soldes restés impayées, les avances faites aux armées françaises et les incessantes réquisitions ${ }^{53}$. Remarquons que, malgré les apparentes bonnes dispositions de Talleyrand ${ }^{54}$, la France ne pouvait songer au rétablissement de la neutralité suisse avant l'époque de la paix générale. 
11 Le 12 nivôse an VIII (2 janvier 1800), Talleyrand informe Zeltner que le gouvernement provisoire « a fait place au gouvernement définitif. Le premier Consul en a la direction éminente " ${ }^{55}$. Par la même occasion, Talleyrand fit part des «sentiments inaltérables de bienveillance et d'attachement » des Consuls " pour un peuple nécessairement ami de la France, son éternel allié par le vœu de la nature, plus étroitement uni encore à elle par l'amour de la liberté ». Après avoir transmis l'intention du gouvernement français «de contribuer au soulagement de votre pays par toutes les mesures que les circonstances rendront possibles à son actif et sincère intérêt ", Talleyrand ouvre un chapitre politique non dénué d'ambiguïté quant à l'avenir de la Révolution en Suisse. Talleyrand annonce ainsi la libération des « otages helvétiques de Bâle, de Berne et de Soleure, détenus, soit à Bitche, soit à Salins ». Et d'ajouter : «Plusieurs de vos notes ont pour objet la situation politique de la Suisse et expriment le vœu de voir la République française vous aider à arriver au mode de gouvernement qui vous assure(ra) le repos et le retour de la sécurité et de la prospérité. Citoyen commissaire, le vœu sincère des Consuls de la République est de voir dans votre pays, rendu au bonheur dont il est digne, la forme de gouvernement voulue par la majorité de vos concitoyens. Les principes que les Consuls de la République font gloire de professer, leur respect religieux pour l'indépendance à jamais inviolable des gouvernements leur défendent l'influence que vous leur avez demandée " ${ }^{56}$. Autrement dit, les partisans de l'Ancien Régime que l'on nomme fédéralistes auront la possibilité de reprendre le pouvoir sans se soucier de l'intervention française pour autant qu'ils se montreront fidèles à la France. Et c'est là que le bât blesse, dans la mesure où les anciens gouvernants, croyant avoir les coudées franches, s'empresseront de renouer des relations privilégiées avec les ennemis de la France, obligeant celle-ci à les renverser à nouveau et à maintenir l'existence cahotique et artificielle de la République helvétique, jusqu'à ce que l'on découvre parmi les patriciens n'ayant rien oublié ceux qui avaient appris quelque chose. Quoi qu'il en soit, en cette fin du XVIII ${ }^{\mathrm{e}}$ siècle, les changements intervenus à Paris en appelaient de comparables en Helvétie où le Directoire helvétique devait pour le moins changer rapidement de nom. Chacun voyait dans le nouveau régime la possibilité qui de rétablir en partie les anciennes institutions, qui d'éliminer les éléments les plus avancés. Au Sénat, le 20 novembre, le Vaudois Jean-Jacques Cart s'en prend à « une constitution qui n'est point notre ouvrage. Elle n'est pas calculée au méridien de l'Helvétie. Elle est sans rapport à nos mœurs, à nos besoins, à nos ressources [...] L'horizon politique s'éclaircit. Un nouveau ciel succède à un ciel nébuleux. Le génie qui alla éclairer l'Orient, a rapporté de l'Orient une vive lumière. Elle éclaire nos espérances; elle promet à l'Europe des sentiments et des principes libéraux. Vous m'entendez citoyens Sénateurs! Empressons-nous d'en profiter et de conduire au port le frêle vaisseau de notre république. Ce port est une bonne constitution " ${ }^{57}$. Fin décembre 1799 un projet d'établissement d'une commission fut aussitôt rejeté par les révolutionnaires de la première heure comme Jean Jacques Cart qui, lors de la discussion au Sénat, en combattit le principe le 31 décembre 1799: "Une première révolution fut suivie en France d'une chaîne de révolutions, une première constitution d'une suite de constitutions. Chacune fut établie en violant la précédente [...] Je vois dans la résolution soumise à votre examen le germe de tous les malheurs qui ont désolé la France. " ${ }^{58}$

4. L'échec des tentatives de La Harpe

12 La Harpe ${ }^{59}$ voulait renforcer le pouvoir de l'Exécutif et proposa une épuration des autorités ainsi qu'une révision très prompte de l'acte constitutionnel afin notamment 
de «détruire le fédéralisme en fondant irrévocablement les diverses peuplades de l'Helvétie ». Et de s'exclamer: "Malheur à qui voudrait entraver notre marche ou arrêter le char de notre révolution " ${ }^{60}$. Dès les 9-10 décembre 1799, il cherche, avec Oberlin ${ }^{61}$ et Secrétan à précipiter les événements et comme l'on disait encore à l'époque à "fructidoriser" la minorité du Directoire et les conseils de la République helvétique. S'appuyant sur la constitution et le traité du 19 août 1798, ils demandent l'ajournement des Conseils et l'appui de la France pour lutter contre l'emprise grandissante du courant réactionnaire. Ce que Étienne Hofmann nomme « une habile manœuvre" déjoue ce projet: "plutôt que de se dissoudre, les Conseils votent la désignation d'une commission de dix membres chargés d'examiner avec le Directoire les mesures à prendre. ${ }^{62}$

13 Le Sénat et le Grand Conseil redoutant de se faire «fructidoriser et brumairiser " ${ }^{63}$ par Laharpe et ses amis nommèrent à la Commission extraordinaire des républicains modérés voire des ennemis de la Révolution comme Aloys Reding, avec l'assentiment de Pichon. Pour Laharpe «ces bruits de fructidorisation et de brumairisation sont autant de calomnies atroces inventées par un parti austro-ologarchique, ennemi juré de la France, partisan déclaré de l'Autriche et voulant à tout prix culbuter le Directoire qui seul, jusqu'ici, a défendu l'alliance avec la République française, et réussi à empêcher les partisans de l'Autriche de faire insurger contre les Français les habitants de l'Helvétie orientale " ${ }^{64}$. Le 6 janvier 1800, Laharpe pressent que le projet du futur gouvernement provisoire issu de la Commission extraordinaire est de faire adopter une constitution « calquée en grande partie sur celle de la France pour tout ce qui tient au Sénat conservateur, qu'on composerait, à l'aide du gouvernement provisoire, des plus fieffés oligarques [...] en un mot de tous ceux qui n'ont cessé d'abhorrer la Révolution française, et de travailler, soit ouvertement soit en secret, contre l'ordre de choses actuel, et en particulier contre la France. » ${ }^{65}$

5. Une conséquence du 18 Brumaire en Helvétie : la journée du 7 janvier 1800

14 L'épreuve de force eut lieu le 7 janvier 1800. La Harpe et ses amis tentent en vain de prendre de vitesse les modérés en invoquant des menées contre-révolutionnaires qui se déroulaient à Berne pour renverser le gouvernement au profit de l'Autriche ${ }^{66}$. Les trois directeurs tentent d'entraîner les militaires français de leur côté en écrivant au général divisionnaire Muller, commandant la division française de l'intérieur : "Les amis de l'Autriche lèvent la tête et veulent renverser le Directoire exécutif, qui, dans tous les temps, fut fidèle observateur de la constitution et de l'alliance française. Dans ces circonstances les membres soussignés vous invitent à prendre pour leur protection et pour celle du gouvernement toutes les mesures auxquelles ils ont le droit d'appeler, en vertu de l'article 3 du traité d'alliance. Le Directoire helvétique vous invite, citoyen général, à faire connaître ces événements au citoyen général en chef Moreau » ${ }^{67}$. Au lieu de répondre Muller s'empressa de joindre Dolder. Privé du soutien français, La Harpe, Secrétan et Oberlin doivent se séparer à 5 heures et demie du soir. Comme peut alors l'écrire le Bulletin helvétique de Lausanne: "La commune de Berne est restée parfaitement tranquille. L'ordre n'a pas été interrompu un instant [...]. Ainsi s'est opérée, sans impulsion étrangère, la révolution du 7 janvier " ${ }^{68}$. Il s'agit bel et bien d'un changement radical, puisque ce 7 janvier 1800 , la Commission des dix fomente en effet un coup d'État, évince La Harpe, Secrétan ${ }^{69}$ et Oberlin. Le Directoire exécutif est aussitôt dissous et aboli, le pouvoir exécutif provisoire étant remis par le Grand Conseil entre les mains de Dolder et Savary ${ }^{70}$. La Harpe ne se faisait guère d'illusion sur Dolder "qu'on verra surnager à toutes les époques " ${ }^{71}$, accusé comme François-Pierre Savary 
(1750-1821) de tenir "pour les plus forts " ${ }^{72}$. Entré au Directoire en juin 1799, Savary est qualifié par La Harpe d'« homme d'esprit mais fin, rusé, intrigant, et qu'on accuse d'être l'âme damnée de l'aristocratie " ${ }^{73}$. À son arrivée à Berne, Pichon trouva Savary et Dolder «qui jouissent dans le pays d'une grande considération, dans les meilleures dispositions » ${ }^{74}$. Les contre-révolutionnaires se réjouirent de la chute de La Harpe : «En abolissant leur directoire exécutif et la constitution de 1798, les réformateurs ont renversé, et avec une prédilection marquée, la fortune politique du Directoire. La Harpe, l'un des plus infatigables, des plus inconsidérés, des plus acharnés promoteurs de la destruction du gouvernement de Berne ${ }^{75}$. De fait, le régime établi par la constitution de 1798 est suspendu. Pour La Harpe, il ne fait aucun doute que la diplomatie française encouragea l'opposition à renverser le Directoire et la constitution unitaire elle-même ${ }^{76}$. Même si le coup d'État du 7 janvier 1800 ne fut pas dirigé par la France, dont l'influence devait se faire sentir discrètement, Pichon déclara à Talleyrand, le 16 janvier, «qu'il avait donné d'avance à ce projet une espèce d'assentiment tacite " ${ }^{77}$. Révélateur est à ce titre l'éloge de Pichon que se croit obligé de faire le gouvernement provisoire dans son premier envoi à Jenner à Paris le 7 janvier : "Le chargé d'affaires Pichon, par la sagesse de sa conduite depuis son arrivée à Berne et en particulier dans ces derniers événements, s'est concilié l'estime, l'affection et la reconnaissance générales. Il a donné à tous les républicains honnêtes de nouveaux motifs de chérir leur alliance avec la France, il leur a donné l'espérance fondée de parvenir par elle à cette tranquillité intérieure et à cette modération de principes dont le peuple à un si grand besoin. » Dolder et Savary s'empressent au passage de charger Jenner de présenter ces événements aux Consuls "comme le résultat profondément senti des besoins $\mathrm{du}$ temps et le fruit $\mathrm{du}$ travail des républicains vertueux et énergiques. Il vous charge de leur représenter d'une manière pressante, de quel intérêt par le succès de cette révolution, de quelle nécessité il serait que le nouveau gouvernement pût s'attacher le peuple, en lui faisant goûter comme fruits de ses premiers travaux et de son union intime avec la République française un soulagement essentiel et général des charges qui pèsent sur l'Helvétie " ${ }^{78}$. C'était alors beaucoup trop demander à la France. Et La Harpe de déplorer en septembre 1801 combien les gouvernements provisoires qui se succédèrent « depuis le 7 janvier 1800, n'étant plus soutenus par l'opinion, tombèrent dans le discrédit, devinrent les instruments de la France, qui s'en servit comme les Anglais en usent avec les Divans et les Nabobs du Bengale " ${ }^{79}$. Cette journée se présente ainsi comme le premier des coups d'État qui jalonneront pendant trois ans cette deuxième période de la République helvétique à son déclin. Le 8 janvier, les deux Conseils (Grand Conseil et Sénat) nomment une Commission exécutive de sept membres, dont feront partie Dolder, Glayre, Finsler, Frisching ${ }^{80}$, Geschwend et Müller, chargé de gouverner jusqu'à la mise en activité d'une nouvelle constitution ${ }^{81}$. Or, des aristocrates comme Frisching, l'ancien trésorier du canton de Berne, préconisaient rien moins que le rétablissement de l'ancienne Confédération sous réserve de quelques arrangements ${ }^{82}$. Les Conseils législatifs entendaient maintenir le principe de la République helvétique et décidèrent le 14 janvier que la nouvelle charte constitutionnelle devait garantir les Droits de l'homme, le principe de l'unité de la République et reposer sur le système représentatif ${ }^{83}$. La Harpe pense que relativement à l'Helvétie, la politique de Bonaparte fut notamment « d'y faire disparaître l'image du gouvernement qu'il venait d'abolir en France et des autorités populaires pour lesquelles il ne parait pas avoir beaucoup d'estime » et «d'affaiblir assez le gouvernement de cette république pour le rendre incapable 
d'aucune opposition à ses volontés " ${ }^{84}$, au point que La Harpe nomma les nouveaux gouvernants "les mannequins du gouvernement provisoire" ${ }^{85}$. À court terme, l'objectif du Premier Consul était la main mise sur le Valais pour s'assurer le contrôle du col du Grand Saint-Bernard qu'il franchira en juin 1800, lors d'un épisode resté célèbre dans les annales militaires. Deux mois et demi plus tard, le 7 septembre 1800, le Premier Consul arrêta l'ouverture de la route du Simplon pour relier la France à la Cisalpine.

15 Privée de base constitutionnelle après cette première violation de la Constitution, la République helvétique dirigée par une Commission exécutive de sept membres, erre désormais sans fondement légal solide et entre pour une durée de trois ans dans une période de profonde incertitude, où toute mesure demeure suspendue et provisoire dans l'attente d'une réforme constitutionnelle voire d'une toute nouvelle constitution, que Bonaparte verrait volontiers beaucoup plus souple. Comme l'a écrit Corinne Chuard: "Tous les ingrédients sont donc réunis pour qu'en l'absence d'une Constitution respectée, la Suisse entre dans une ère de divisions et de conflits internes ${ }^{86}$. Dans une lettre du 17 juin 1800, adressée à un La Harpe désabusé, impatient de se retirer en France, Peter Ochs se dira convaincu « qu'il y a encore moyen de sauver la révolution" ${ }^{87}$. La bonne volonté ne manquait pas aux nouvelles autorités helvétiques. Dès le 27 janvier 1800, elles avaient fait part au Premier Consul de leur souhait de voir la paix garantir "à l'Helvétie son antique indépendance. Jamais aucun peuple n'en ressentit avec plus de douleur la perte " ${ }^{88}$. Le 8 août 1800 , le pouvoir législatif (Sénat et Grand Conseil) est dissous pour avoir résisté aux pressions françaises et remplacé par un Conseil législatif. La Commission exécutive prend le nom de Conseil exécutif.

6. L'idée de médiation

16 Entre 1800 et 1802 il y eut quatre coups d'État et cinq projets constitutionnels. Les Français s'efforcèrent de maintenir un régime provisoire jusqu'à l'heure du règlement définitif de la question suisse dans le cadre de la pacification générale sur le continent. Le Premier Consul n'eut de cesse d'obtenir une Suisse pacifiée, raisonnablement affaiblie et tributaire de la France. Pour cela il fallait achever la Révolution, substituer à l'étape unitaire une vision fédéraliste. Le Consulat privilégia de plus en plus ouvertement les hommes de l'Ancien Régime. Pour La Harpe, Reinhard, l'envoyé de France en Suisse « réussit à les enjôler en leur dépeignant le gouvernement consulaire de France comme l'ennemi des principes démocratiques en Suisse. Ils le crurent » ${ }^{89}$. Ce soubassement fédératif renouvelé ne signifiait en aucun cas le retour aux villes-cantons centralisées d'avant 1798 qui aurait signifié la restauration des États aristocratiques et donc de l'hégémonie bernoise acquise aux ennemis de la France nouvelle formule. En cherchant avant tout à ménager les intérêts stratégiques de la France, il a su ainsi trouver pour la Suisse un compromis acceptable entre les aspirations nouvelles et les forces traditionnelles. Ce faisant, Napoléon Bonaparte ne faisait que poursuivre la politique ancestrale de la France. Sous l'Ancien Régime, le roi de France fonctionnait déjà tel un Médiateur entre les différentes parties de la Suisse sans en posséder formellement le titre. Bonaparte fut très tôt appelé à jouer ce rôle. Dès 1797, pour imposer le régime unitaire, le Bâlois Peter Ochs (1752-1821) sollicita l'intervention de Bonaparte, l'invitant même à se faire «le législateur de la Suisse ", ce que le général refusa d'être ${ }^{90}$. 
17 Par une loi du 13 janvier 1800, il fut décidé par les Conseils législatifs l'élaboration d'une nouvelle constitution unitaire et représentative. Une nouvelle constitution devait être mise en place que cherchèrent à influencer les deux principaux partis en présence. Le parti unitaire voulait le maintien du gouvernement central dans toute sa plénitude, tandis que le parti fédéraliste, composé entre autres des députés des petits cantons, désirait le rétablissement des anciens cantons avec leurs droits territoriaux et souverains. Finalement, la constitution dite de la Malmaison adoptée par le corps législatif, le 29 mai 1801 servit de matrice aux constitutions à venir. Pour imposer ses vues, le Premier Consul réunit une Consulta helvétique qui se déroula entre décembre 1802 et janvier 1803. On se souvient du mot de Reubell : « Il faut croquer les petits pâtés suisses ». Napoléon sut mettre en application ce conseil ${ }^{91}$ de façon judicieuse. Car enfin, « il s'agissait, en effet, de faire octroyer une constitution à un peuple théoriquement libre et souverain par un chef de gouvernement étranger $"{ }^{92}$. Occasion pour Napoléon qui perçait sous Bonaparte de signifier clairement au fondateur de la République helvétique : «Monsieur Ochs, la Révolution est finie. »

Le 29 janvier 1803, une ultime conférence se déroula aux Tuileries, sur les lieux mêmes où un peu plus de dix ans plus tôt les Gardes-suisses se sacrifiaient pour la sauvegarde de la monarchie. "Napoléon déploya la plus rare connaissance des affaires suisses et des besoins divers des cantons; il chercha à convaincre les unitaires de la nécessité du maintien des landsgemeinden et les fédéralistes de l'impossibilité du rétablissement des privilèges. Il discuta sept heures durant, avec une éloquence admirable, toutes les grandes questions relatives au nouveau pacte qu'il se proposait de donner à la Suisse. Puis, le 19 février suivant, réunissant tous les députés dans une séance solennelle aux Tuileries, il remit l'Acte de médiation au député de Fribourg, Louis d'Affry, désigné par le premier consul pour occuper le poste éminent de Landamman de la Suisse, créé par la nouvelle constitution ${ }^{93}$. L'Acte de médiation est considéré par Michel Vovelle comme un coup de génie : «Il a su traiter avec les républicains les plus modérés et les notables pour donner à la Suisse une ébauche de structure fédérale qu'on retrouvera en partie en 1848. En grand manipulateur, le général avait bien compris votre pays. C'est d'ailleurs la dernière intervention politique de la France en Suisse. Napoléon avait repris à son compte le rôle séculaire des rois de France. » ${ }^{94}$

19 Napoléon Bonaparte a donné à la Suisse une assise politique et cantonale stable, en jetant les bases d'un État fédéraliste moderne qui a permis la coexistence de régimes politiques très divers. En établissant parallèlement la dignité et l'égalité des États cantonaux, la Médiation a permis à ceux-ci d'entamer leur intégration à un État national, et d'évoluer en partant de leur situation historique et selon des rythmes et des modalités spécifiques en faisant abstraction des structures et tares héritées de la période d'assujettissement des territoires récemment émancipés. On remarquera d'ailleurs que les territoires des 19 cantons n'ont depuis lors pratiquement pas subi de modifications, hormis le cas bernois. De même, à travers la personnalité du Landamman de la Suisse, se profile l'idée d'une autorité centrale permanente, tandis que la Diète était désormais seule compétente pour la politique extérieure tout en délibérant à la majorité. Cet Acte que l'on a également désigné sous le nom évocateur de petite Restauration rompt en effet avec le principe de l'unité et renoue avec la tradition fédérative de l'ancien Corps helvétique. Il donne un avant-goût de la nature même du régime impérial qui sera instauré l'année suivante. 
Comme le rappelle Strickler, la Suisse fut pour la France " ein Ring am kleinen Finger ${ }^{95}$. Et Jean-René Suratteau d'ajouter: "Les barreaux de la cage étaient soigneusement camouflés, mais ils n'en étaient que plus solides " ${ }^{96}$. La Suisse de la Médiation cachait sous son apparente autonomie une "réelle nullité» ${ }^{97}$. Authentique "satellite de l'Empire napoléonien ", elle devenait au grand jour ce qu'elle n'avait pas manqué d'être dans les faits à maintes occasions depuis Louis XIV.

Bonaparte se montra très clair: "L'obscurité politique vous convient uniquement " ${ }^{98}$ affirma Bonaparte lors de son allocution du 29 janvier 1803, avant de préciser au patricien bernois Watteville: "Je ne souffrirai jamais aucune autre influence en Suisse que la mienne, dût-il m'en coûter cent mille hommes " ${ }^{99}$. Comme l'a exprimé Charles Monnard: "L'autorité suprême de l'Helvétie venait de passer ostensiblement à Paris. Son gouvernement ne possédait plus qu'une autorité nominale " ${ }^{100}$. Pour le futur empereur, la neutralité suisse était un mot vide de sens en dehors de lui. Comme avait pu l'écrire le savant et diplomate bernois Philippe-Albert Stapfer: "ce serait une illusion que de se bercer de l'espoir de jamais obtenir pour la Suisse autre chose que ce qui sera conforme aux intérêts du gouvernement français. » ${ }^{101}$

\section{NOTES}

1.Voir à ce sujet notre texte : "L'invasion tardive des cantons suisses en 1798 » in 115e, 116e congrès national des sociétés savantes, 1990-1991, Tome 1, Commission d'histoire de la Révolution française, pp.115-120.

2.Voir l'article «Jean-Frédéric Perregaux banquier (1744-1808 » de Thierry CLAEYS et Alain-Jacques TORNARE in : Biographies neuchâteloises, Hauterive, Éd. Gilles Attinger, 1996, pp. 207-211. Rappelons ici que le troisième siège de régent, le seul qui pendant près d'un siècle et demi allait passer sans interruption de père en fils est celui du banquier genevois Mallet. Signalons également la présence dans l'entourage de Napoléon Bonaparte du Bernois Haller, l'ancien associé de Necker, munitionnaire et trésorier de l'armée d'Italie. Sur la plupart des personnages suisses cités ici, on peut consulter les toutes récentes biographies à paraître dans le Dictionnaire Historique de la Suisse et consultables pour la plupart d'entre elles sur le site Internet : www.dhs.ch 3.Actensammlung aus der Zeit der helvetischen Republik (1798-1803), Bearbeitet von Johannes Strickler, V. Band, Bern, 1895, p. 519, n 218. Motion du 7 janvier 1800. 4.En dehors de la période révolutionnaire son nom est Frédéric-César de La Harpe. 5.Actensammlung..., op.cit., pp. 219, $\mathrm{n}^{\circ} 96$.

6.Correspondance de Frédéric-César de la Harpe sous la République helvétique, publiée par Marie-Claude JÉQUIER, tome III, Éditions Slatkine, Genève, 1998, p. 391, n 512. AN, 284 AP 13 , dossier 12 .

7.Id., p. 414, n 528. AN, Papiers Sieyès, 284 AP 13, dossier 12.

8.Introduction à la Correspondance de Frédéric-César de la Harpe sous la République helvétique, publiée par Marie-Claude JÉQUIER. 
9.Actensammlung..., op. cit., pp. 496-497, n² 210/8. Lettre du Directoire helvétique à Zeltner, 20 novembre 1799.

10.Correspondance de Frédéric-César de La Harpe et Alexandre ler, op. cit., tome 1, $\mathrm{n}^{\circ}$ 68, $\mathrm{p}$. 277 , ler octobre 1801.

11.Id., p. 266, $\mathrm{n}^{\circ}$ 68. Lettre du ler octobre 1801. Sur le Directoire de Suisse voir notre contribution : «L'image de la France du Directoire au travers des récits de contemporains suisses » in La République directoriale, Clermond-Ferrand, 1997, vol. 2, pp. 961-986.

12.B. NABONNE, « La diplomatie du Directoire d'après les papiers inédits de Reubell », Paris, 1951, p. 127.

13.Rapinat, beau-frère de Reubell, symbolise en Suisse la conquête spoliatrice.

14.AAE, CP, Suisse, CCCLXX, 197.

15.Alfred RUFER, « La République helvétique », reproduit par J.-R. SURATTEAU, La Suisse et la Révolution française, Paris, 1973, p. 37. Sur le thème de la Suisse ménagée par la Révolution on peut consulter notre communication : "L'invasion tardive des Cantons Suisses en 1798 » in Actes des 115e-116e congrès national des sociétés savantes, 1990-1991, Tome 1, pp. 115-130.

16. Mallet du Pan utilise cette formule dans son Mercure Britannique, $\mathrm{n}^{\circ}$ XXXIV, du 10 février 1800 , p. 36.

17.Jacques MALLET, dit MALLET DU PAN (1749-1800), publiciste génevois, dirigea à Paris le Mercure historique et politique de Genève (1783-1788); ennemi de la Révolution française, il rentre à Genève puis se réfugie en Angleterre.

18. Mercure Britannique, $\mathrm{n}^{\circ} \mathrm{XXIV}, \mathrm{p} .34$.

19.Id., $\mathrm{n}^{\circ}$ XXIX, 25 novembre 1799 : « Nouvelle Révolution du 9 de ce mois », p. 24.

20.Id., $\mathrm{n}^{\circ}$ XXVIII, 10 novembre 1799, p. 36.

21.Id., $\mathrm{n}^{\circ}$ XXIX, 25 novembre 1799 : « Nouvelle Révolution du 9 de ce mois », p. 25.

22.Id., $\mathrm{n}^{\circ}$ XXXI, 25 décembre 1799, p. 26. Voir également le no XXXII du 10 janvier 1800 , pp. 11-32 : «France. Observations générales sur le nouveau système législatif de cette république $»$.

23.Id., $\mathrm{n}^{\circ} \mathrm{XXXV}, 10$ mars 1800 , pp. 28 et 34.

24.Id., $\mathrm{n}^{\circ}$ XXXVI, 25 mars 1800, p. 36.

25.Id., $\mathrm{n}^{\circ} \mathrm{XXXV}, 10$ mars 1800, pp. 21-22.

26.Actensammlung..., op.cit., S. 478-479, $\mathrm{n}^{\circ} 208 / 5$.

27.Émile DUNANT : Les relations diplomatiques de la France et de la République Helvétique, Basel 1901. Quellen zur schweizer Geschichte, S. LXIX.

28.Secrétaire de la légation française à Berne du 9 novembre 1799 au 23 février 1800, jour de l'arrivée de Reinhard.

29.AAE, Paris, Correspondance diplomatique, fonds Suisse, Tome $471, \mathrm{n}^{\circ} 114$. É. DUNANT, ouvr. cit., p. $274, \mathrm{n}^{\circ} 830$.

30. Correspondance La Harpe-Alexandre Ier, op. cit., tome 1, $n^{\circ} 68$, p. 277, ler octobre 1801. 31.Après la révolution du 7 janvier 1800, Zeltner fut remplacé par Amédée Jenner, comme ministre helvétique à Paris.

32.Ministre des Relations extérieures jusqu'au 22 novembre 1799, il fut ministre plénipotentiaire en Suisse du 22 février 1800 au 7 septembre 1801 . Voir à ce sujet les Actensammlung..., op. cit., pp. 826-829.

33.Actensammlung..., op. cit., p. 495, $\mathrm{n}^{\circ} 210 / 4$. Sur le 18 Brumaire et ses suites en Suisse voir pp. 494-502. 
34.Gottlieb Abraham von Jenner (1765-1834), patricien bernois, chargé d'affaires de la République helvétique à Paris (juin 1798-septembre 1800).

35.BCU, Lausanne, Fonds La Harpe, J 156, 201. Correspondance de Frédéric-César de la Harpe sous la République helvétique, publiée par Marie-Claude JÉQUIER, tome III, Éditions

Slatkine, Genève, 1998, p. 482, $\mathrm{n}^{\circ} 568$.

36. BCU, Lausanne, Fonds La Harpe, H 33, 208. Corr. Laharpe, $n^{\circ} 566$, p. 479. Voir aussi sa lettre à de Bry du 10 août 1798. Corr. Laharpe, $n^{\circ} 362$.

37.AN, Papiers Sieyès, 284 AP 16, dossier 2 (5 bis). Correspondance de Frédéric-César de la Harpe sous la République helvétique, op. cit., tome III, Éditions Slatkine, Genève, 1998, p. $485, \mathrm{n}^{\circ} 569$.

38. Voir à ce sujet sa lettre à Sieyès du 6 juin 1799 et son mémoire adressé de Berne le 5 juillet 1799. AN, 284 AP 13, dossier 12. Correspondance de Frédéric-César de la Harpe sous la République helvétique, op. cit., tome III, Éditions Slatkine, Genève, 1998, p. 391-392; 414-420, nos 512 et $528, \mathrm{n}^{\circ} 569$.

39.AN, AF IV , 1701, Dossier 3, $\mathrm{n}^{\circ} 1$. Correspondance de Frédéric-César de la Harpe sous la République helvétique, op. cit., tome III, Éditions Slatkine, Genève, 1998, p. 519, nº 585.

40.Korrespondenz des Peter Ochs, Hrg. Von Gustav Steiner, Basel, 1935, II, p. 627. Jenner à Zerleder, Paris, 21 juin 1799.

41.Correspondance de Frédéric-César de la Harpe sous la République helvétique, op. cit., tome III, Éditions Slatkine, Genève, 1998, pp. 501-511, nº 580.

42.Henri-François Constance Perrochel (1751-1810).

43. Correspondance La Harpe-Alexandre Ier, op. cit. tome 1, $\mathrm{n}^{\circ} 68, \mathrm{p} .277$, ler octobre 1801. 44.Étienne HOFMANN, préface à la Correspondance de Frédéric-César de la Harpe sous la République helvétique, op. cit., tome III, Éditions Slatkine, Genève, 1998, p. 31. AAE, Paris, Correspondance diplomatique, fonds Suisse, Tome 471, $\mathrm{n}^{\circ} 125$ à 153 . Voir à ce sujet Emile Dunant, Les relations diplomatiques de la France et de la République helvétique : 1798-1803, Bâle 1901, Quellen zur Schweizer Geschichte, 19, pp. 276-282.

45.Étienne HOFMANN, préface à la Correspondance de Frédéric-César de la Harpe sous la République helvétique, op. cit., tome III, Éditions Slatkine, Genève, 1998, p. 31.

46.Id., p. 11.

47. Rapport du ministre Talleyrand au ler Consul, mi-janvier 1800. op. cit. v. p. 830.

48.É. DUNANT, op. cit., $\mathrm{n}^{\circ}$ 851, p. 283. Pichon à Zalleyrand, 12 frimaire an VIII.

49.Voir à ce sujet le texte d'Évelyne MARADAN : «L'Échec de la propagande du Club helvétique de Paris auprès du régiment des Gardes 1789-1791 » in Paris et la Révolution, Publications de la Sorbonne, Paris, 1991, pp. 253-267.

50.Actensammlung..., op. cit., p. 498, $\mathrm{n}^{\circ} 210 / 10$.

51.Actensammlung..., op. cit., p. $499, \mathrm{n}^{\circ} 210 / 13$.

52.É. DUNANT, op. cit., $\mathrm{n}^{\circ} 840$, p. 278.

53.Actensammlung..., op. cit., p. 496, $\mathrm{n}^{\circ} 210 / 5$.

54.Voir à ce sujet le rapport de Talleyrand aux Consuls du 30 frimaire an VIII. AAE, Paris, Correspondance diplomatique, fonds Suisse, Tome 471, $\mathrm{n}^{\circ} 193$. Cf. É. DUNANT, op. cit., $\mathrm{n}^{\circ} 870$, pp. 293-295.

55.Actensammlung..., op. cit., p. 501, n² 210/18.

56.Id., p. 502, $\mathrm{n}^{\circ}$ 210/19.

57.Bulletin officiel vaudois, $X, 179,180$. Actensammlung..., op. cit., V, n² 235, p. 615.

58.Actensammlung..., op. cit., p. 489, $\mathrm{n}^{\circ}$ 208/16.

59.Nommé membre du Directoire helvétique le 29 juin 1799, La Harpe a été en fonction jusqu'au 7 janvier 1800 . 
60.Actensammlung..., op. cit., p. 225.

61.Victor Oberlin (1747-1818), membre et président du gouvernement soleurois en 1798, Directeur helvétique (avril 1798-janvier 1800).

62.Préface d'Étienne Hofmann à la Correspondance de Frédéric-César de la Harpe sous la République helvétique, op. cit., tome III, Éditions Slatkine, Genève, 1998, p. 31.

63.Selon la formule de Laharpe dans une lettre à Zeltner du 2 janvier 1800. Archives de l'État de Berne, Briefe an Zeltner, Bs 1. Correspondance de Frédéric-César de la Harpe sous la République helvétique, op.cit., tome III, Éditions Slatkine, Genève, 1998, p. 541, n 595.

64.Lettre au rédacteur du Moniteur, Correspondance de Frédéric-César de la Harpe sous la République helvétique, op.cit., tome III, Éditions Slatkine, Genève, 1998, p. 542.

65.Lettre de Laharpe à Zeltner, 6 janvier 1800, Archives de l'État de Berne, Briefe an Zeltner, BS 1. Correspondance de Frédéric-César de la Harpe sous la République helvétique, op. cit., tome III, Editions Slatkine, Genève, 1998, p. 544, nº 596.

66.Sur les ingérences autrichiennes en Suisse, voir notre contribution en collaboration avec Evelyne Maradan : « Les tentations autrichiennes en Suisse durant la Révolution française » in Austriaca, décembre 1989, n² 29, pp. 61-83.

67.Actensammlung..., op. cit., $\mathrm{n}^{\circ} 219$, p. 523.

68.Id., n² 220, p. 539. Bulletin helvétique XI, 71, 72.

69.Philippe Secretan (1756-1826), de Lausanne, avocat, président de l'Assemblée constituante en 1798, membre du Directoire helvétique de 1799 à 1800 .

70.Johann Rudolf DOLDER (1753-1807), homme politique zurichois et argovien, membre du Directoire 1799-1800, puis de la Commission exécutive, landamman de la République helvétique. François-Pierre SAVARY (1750-1821), patricien et médecin fribourgeois, de tendance fédéraliste, membre du Directoire helvétique (1799-1800), de la Commission exécutive (1800-1801). Une note française sur le personnel politique helvétique relève à son sujet : « Mouche de coche, aimant à protéger les hommes de qualité. De plus Bigot », mais « attaché à la France ». AN, AF IV 1701.

71.BCU, Lausanne, Fonds La Harpe, G Aa 17. Annotation de La Harpe dans son « Précis historique sur la Révolution helvétique » adressé à Alexandre ler, du ler octobre 1801. Correspondance de Frédéric-César de La Harpe et Alexandre Ier, op.cit., tome 1, p. 288.

72.Ibid., G Aa 13. Note à une lettre du 30 août 1801. Correspondance de Frédéric-César de La Harpe et Alexandre Ier, Ibid., p. 247.

73.Ibid., H 33, 197. Correspondance de Frédéric-César de la Harpe sous la République helvétique, III, $\mathrm{n}^{\circ}$ 523, p. 404.

74.AAE, CP, Suisse, CCCCLXXI, 153, 19 Brumaire an VIII.

75. Mercure Britannique, 10 mars 1800, $\mathrm{n}^{\circ} \mathrm{XXXV}$, p. 21.

76.Correspondance de Frédéric-César de La Harpe et Alexandre Ier, op. cit., tome ler, p. 233, $\mathrm{n}^{\circ}$ 62, lettre à Alexandre ler du 27 germinal an IX/17 avril 1801. Voir aussi p. 238.

77.É. DUNANT, op. cit., pp. LXI-LXII.

78.Actensammlung..., op. cit., III, $\mathrm{n}^{\circ} 221$, p. 540.

79. Correspondance de Frédéric-César de La Harpe et Alexandre Ier, op.cit., p. 252, nº 67.

80.Signe des temps, le patricien bernois Frisching représentait les anciens gouvernants. 81.Actensammlung..., op. cit., p. 545, $\mathrm{n}^{\circ} 225$.

82.AAE, Paris, Correspondance diplomatique, fonds Suisse, Tome 471, $\mathrm{n}^{\circ} 174$. É. DUNANT, op. cit., $\mathrm{n}^{\circ} 860$, p. 287. Pichon à Talleyrand, 18 frimaire an VIII.

83.Actensammlung..., op. cit., p. 272, $\mathrm{n}^{\circ} 235$.

84. Correspondance de Frédéric-César de La Harpe et Alexandre Ier, op. cit., tome 1, $\mathrm{n}^{\circ}$ 68, $\mathrm{p}$. 278, 1er octobre 1801. 
85.Id., $\mathrm{n}^{\circ} 68$, p. 269.

86. Corinne CHUARD, 1798 : à nous la liberté. Chronique d'une révolution en Pays de Vaud, 24

Heures, 1998, p. 222.

87. Korrespondenz des Peter Ochs, Hrg. Von Gustav Steiner, II, $\mathrm{n}^{\circ} 4$, p. 4.

88. Archives Fédérales, Berne, Helvétique 792, p. 67-68. Actensammlung... op. cit., V, p. 702.

89. Correspondance de Frédéric-César de La Harpe et Alexandre ler, op. cit., p. 259, n 67.

90.Ochs à Bonaparte, 21 nivôse, ler décembre 1798; Correspondance Panckoucke, IV, 471. Le même au même, 7 ventôse. AN, AF IV, 1049.

91.Jean-René SURATTEAU, « La Suisse dans le système français, 1799-1813 » in Les pays sous domination française, 1968, p. 96.

92.William RAPPARD, L'individu et l'État, p. 67.

93.Alexandre DAGUET, Histoire de la Confédération suisse, Lausanne, 1865, p. 520.

94.Cf. La Liberté, lundi 22 juin 1998, p. 24. Interview accordé à Patrick Vallélian.

95.Johannes STRICKLER, « Der französisch schweizerische Handelsvertrag vom 30. Mai

1795 ». Politisches Jahrbuch, 1892, p. 291.

96.Jean-René SURATTEAU : «La Suisse dans le système français, (1799-1813) », op. cit., p.

104.

97.Selon la formule de Jean-René SURATTEAU, id., p. 93.

98.Archives Fédérales, Berne. Archives helvétiques, $n^{\circ} 3361, n^{\circ} 595$.

99.Mutach's Revolutionsgesch.msc II, 288-290.

100. Charles MONNARD, Histoire de la Confédération suisse par Jean de Müller....traduite de l'allemand et continuée jusqu'à nos jours par MM. Charles Monnard et Louis Vuillemin, ParisLausanne 1847, XVII, p. 326.

101.Bonaparte, Talleyrand et Stapfer 1800-1803, Zurich, 1869, p. 13.

\section{RÉSUMÉS}

Lorsque survient la journée du 18 Brumaire, une partie de la Suisse sert de champ de bataille aux grandes puissances et se trouve encore occupée par les forces de la coalition. En cet automne 1799, la misère et l'épuisement de la Suisse étaient tels que le désespoir touchait toutes les couches de la population. Les relations entre le Directoire français et son homologue helvétique étaient alors particulièrement tendues. Tous les partis en Suisse accueillirent ou firent semblant d'accueillir le 18 Brumaire avec soulagement. À commencer par les adversaires de la Révolution qui conçurent l'espoir d'être à l'avenir soutenu par les Consuls de la République française. Les changements intervenus à Paris en appelaient de comparables en Helvétie où le Directoire helvétique sera dissous le 7 janvier 1800. Avec le départ du patriote vaudois Frédéric-César Laharpe, la République helvétique entre dans une phase de décadence inexorable. Les Conseils législatifs chargés d'établir une nouvelle constitution cherchèrent en vain à obtenir le rétablissement de la neutralité, l'allégement du poids de l'occupation française, et une réforme du recrutement des troupes auxiliaires helvétiques. Du point de vue suisse le 18Brumaire ouvre une époque de déstabilisation des institutions politiques issues de la Révolution helvétique. 
The Response to 18 Brumaire in Helvetia. When the coup of 18 Brumaire occurred, part of Switzerland served as a battle-field to the grest powers and was still occupied by coalition forces. The wretchedness and exhaustion of Switzerland in the autumn of 1799 were such that all sections of the population were subject to despair. Relations between the French Directory and its Swiss counterpart were then severely strained. All parties in Switzerland welcomed or appeared to welcome the coup with relief, especially the opponents of the Revolution who fondly hoped to win the future support of the Consuls of the French Republic. The changes occurring in Paris resulted in comparable changes on the Swiss side, with the Helvetic Directory being dissolved on 7 January 1800. With the departure of the Vaudois patriot Frédéric-César Laharpe, the Helvetic Republic entered a phase of inexorable decline. The legislative Councils tasked with establishing a new constitution sought in vain a restoration of neutrality, relief from the burden of French occupation and changes to the custom of levying Helvetic auxiliaries. From the Swiss viewpoint, 18 Brumaire ushered in a period of destabilisation of the politicai institutions born with the Helvetic Republic.

L'accoglienza del 18 Bruamaio nella regione elvetica. Quando arriva il 18 Brumaio, una parte della Svizzera funge da campo di battaglia per le grandi potenze e si trova ancora occupata dalle forze della Coalizione. In qusto autunno 1799 la miseria e lo sfinimento della Svizzera erano tali che la disperazione colpiva tutti gli strati sociali. Le relazioni tra il Direttorio francese e il suo omologo elevetico erano in quel momento particolarmente tese. Tutti i partiti in Svizzera accolsero o finsero di accogliere il 18 Brumaio con sollievo. A cominciare dagli avversari della Rivoluzione che concepirono la speranza di essere per il fututo sostenuti dai Consoli della Repubblica francese. I camblamenti intervenuti a Parigi ne richiamavano altri in Svizzera, dove il Direttorio elevetico sarà sciolto il 7 gennaio 1800. Con la partenza del patriota valdese FrédéricCésar Laharpe, la Repubblica Elvetica entra in una fase di inesorabile decadenza. I Consigli legislativi incaricati di stabilire una nuova costituzione cercarono invano di ottenere il ristabilimento della neutralità, l'alleggerimento del peso dell'occupazione francese e una riforma del reclutamento delle truppe ausiliarie elvetiche. Dal punto di vista svizzero il 18 Brumaio apre un'epoca di destabilizzazione delle istituzioni politiche nate dalla Rivoluzione elvetica.

Cuando el 18 Brumaire, una parte de Suiza esta un camp de batalla para las grandes potencias, y se encuentra todavía ocupada por las fuerzas de la coalición. Durante este otoño de 1799, la miseria y el agotamiento de Suiza explican la desesperación de todas las capas de la población. Las relaciones entre el Directorio frances y su homólogo helvético estaban muy tirantes. Todos los partidos en Suiza acogeron o simularon de acoger el 18 Brumaire con alivio. Los adversarios de la Revolución esperaban el apoyo de los consules de la República francesa. Los cambios en París suscitaron cambios en Helvecia donde el directorio fue disuelto el 7 e enero de 1800. Con la salida del patriota valdense Fréderic-César Laharpe, la república helvética entra en una fase de decadencia inexorable. Los consejos legislativos, cargados de establecer una nueva constitución, buscaron en vano el restablecimiento de la neutralidad, la disminución del peso de la ocupación francesa, y una reforma del reclutamiento de las tropas auxiliares helvéticas. Visto desde Suiza, el 18 Brumaire abre une époqua de desestabilización de las instituciones políticas nacidas de la revolución helvética.

Die Rezeption des 18. Brumaire in Helvetien. Als der Staatsstreich in Frankreich stattfindet, dient ein Teil der Schweiz als Schachtfeld für die großen Mächte und ist immer noch von den Streitkräften der Koalition besetzt. In diesem Herbst 1799 waren das Elend und die Erschöpfung der Schweiz so groß geworden, daß die Verzweiflung alle Schichten der Bevölkerung ergriffen hatte. Alle Parteien empfingen oder taten, als empfingen sie den 18. Brumaire mit Erleichterung. Dies schon bei den Gegnern der Revolution, die die Hoffnung hegten, in der Zukunft von den französischen Konsuln unterstützt zu werden. Die politischen Änderungen in Paris zwangen die 
Schweiz, einen ähnlichen Weg einzuschlagen. Folglich wurde das helvetische Direktorium am 7. Januar 1800 aufgelöst. Nach dem Weggang des waatländischen Patrioten Frédéric-César Laharpe begann für die helvetische Republik eine Zeit des unwiderstehlichen Verfalls. Die gesetzgebenden Räte, die den Auftrag hatten, eine neue Verfassung zu entwerfen, versuchten vergeblich, die Wiederherstellung der Neutralität, eine Erleichterung der Lasten der französischen Besatzung und eine Reform der Rekrutierung der helvetischen Hilfstruppen zu erlangen. Vom schweizerischen Standpunkt aus gesehen beginnt mit dem 18. Brumaire eine Periode der Entstabilisierung der aus der helvetischen Revolution herrührenden politischen Einrichtungen.

\section{AUTEUR}

\section{ALAIN-JACQUES CZOUZ-TORNARE}

Université de Fribourg 